Utz von Wagner, Lukas Lentz

\title{
On the detection of artifacts in Harmonic Balance solutions of nonlinear oscillators
}

Conference paper | Accepted manuscript (Postprint)

This version is available at https://doi.org/10.14279/depositonce-9832

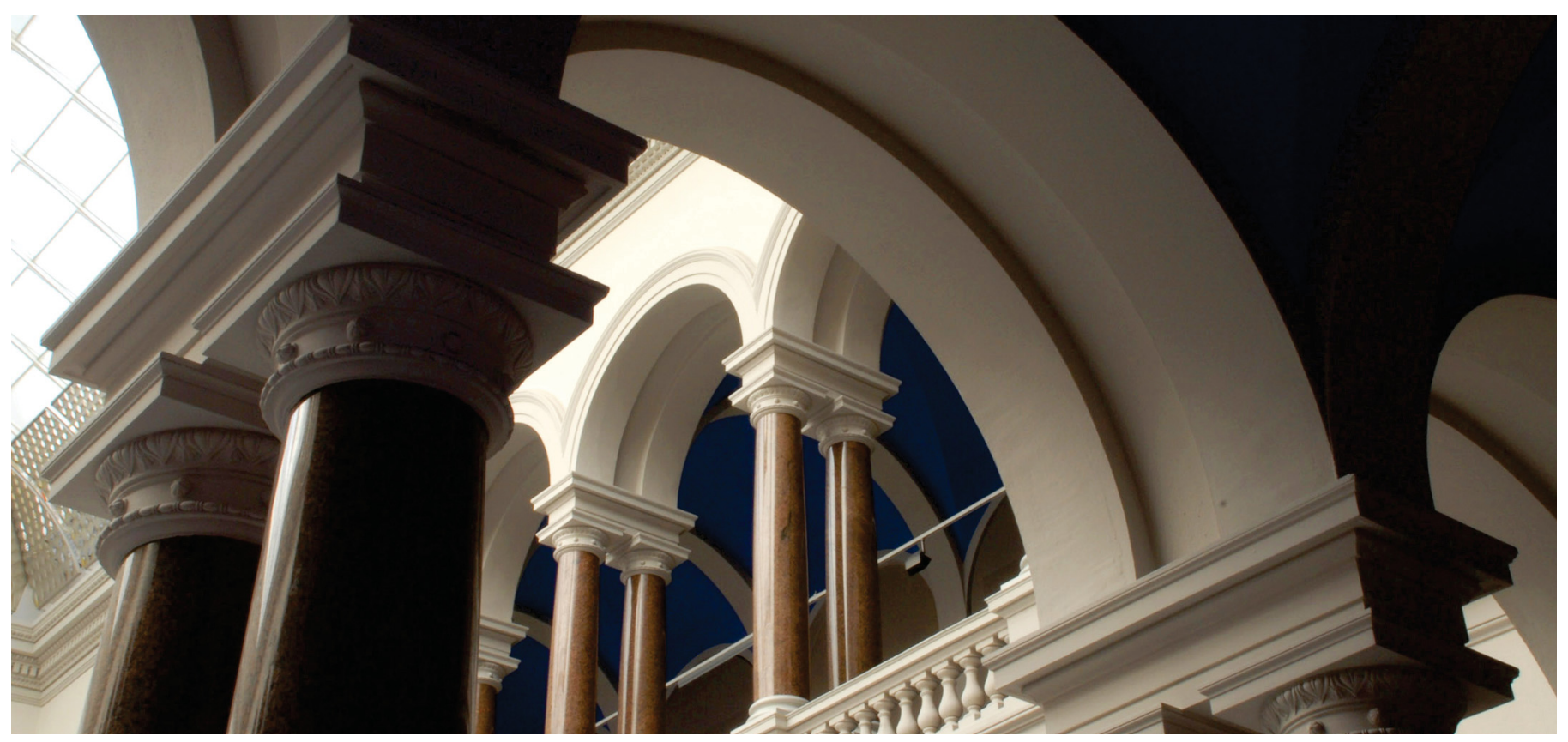

von Wagner, U., \& Lentz, L. (2019). On the detection of artifacts in Harmonic Balance solutions of nonlinear oscillators. Applied Mathematical Modelling, 65, 408-414. https://doi.org/10.1016/j. apm.2018.08.013 


\title{
On the Detection of Artifacts in Harmonic Balance Solutions of Nonlinear Oscillators
}

\author{
U. von Wagner ${ }^{\mathrm{a}, *}$, L. Lentz ${ }^{\mathrm{a}}$ \\ ${ }^{a}$ Institut für Mechanik, Technische Universität Berlin, Einsteinufer 5, 10587 Berlin, Germany
}

\begin{abstract}
Harmonic Balance is a very popular semi-analytic method in nonlinear dynamics. It is easy to apply and is known to produce good results for numerous examples. Adding an error criterion taking into account the neglected terms allows an evaluation of the results. Looking on the therefore determined error for increasing ansatz orders, it can be evaluated whether a solution really exists or is an artifact. For the low-error solutions additionally a stability analysis is performed which allows the classification of the solutions in three types, namely in large error solutions, low error stable solutions and low error unstable solution. Examples considered in this paper are the classical Duffing oscillator and an extended Duffing oscillator with nonlinear damping and excitation. Compared to numerical integration, the proposed procedure offers a faster calculation of existing multiple solutions and their character.

Declarations of interest: none.
\end{abstract}

Keywords: Nonlinear dynamics, Harmonic Balance method, Duffing oscillator

\section{Introduction}

The investigation of nonlinear dynamical problems in Mechanics and Mechatronics is of permanent interest. For investigating basic properties of such systems, often simple nonlinear oscillators with one degree of freedom are considered. There are several semi-analytic methods known for solving such problems which are described in textbooks e.g. by Hagedorn [1]. Among them, Harmonic Balance is an easy to apply method, which is very popular even in actual publications, sometimes compared with other methods like normal form transformation or multiple scales e.g. [2]. Harmonic Balance is combined in the present paper by a corresponding error criterion which is an extension of the error criterion introduced by Urabe et al. [3]. Additionally a stability analysis is performed for the low error solutions. Another actual attempt for

\footnotetext{
${ }^{*}$ Corresponding author. Tel: +49 30 31421169; Fax: +49 3031421173

Email addresses: utz.vonwagner@tu-berlin.de (U. von Wagner), L.Lentz@tu-berlin.de (L. Lentz)
} 
improvements or additions to the Harmonic Balance method is e.g. the reduction of the resulting systems of nonlinear algebraic equations by neglecting higher order terms [4], [5]. In the Incremental Harmonic Balance method, a variable number of harmonic functions is used to ensure a defined accuracy of the solution [6], [7]. The identification of non-physical solutions, later called in this paper artifacts, is not in the focus of these publications.

Among the examples of academic nonlinear oscillators, the Duffing oscillator is very popular [8]. Therefore as examples a classical Duffing oscillator with solely cubic nonlinearity and an extended Duffing oscillator are chosen. Compared with the papers of the same authors [9], [10] and [11] where the same (and other) examples are studied, the parameters investigated in this paper have been changed also resulting in other phenomena and qualitative behavior. Furthermore a convergence analysis has been added.

\section{Classical Duffing oscillator}

Our studies focus in this section on the softening Duffing oscillator where solutions being suspicious for artifacts are obtained by Harmonic Balance [9]. As in [9], [10] and [11] additionally to the Harmonic Balance, an accompanying error criterion is applied which is an extension of the error criterion introduced by Urabe et al. [3]. As examples for the application and further development of the criterion by Urabe et al., beside the already mentioned work of the authors, [12] and [13] shall be mentioned.

The softening Duffing oscillator is used in the following for the introduction of the method and to show some additional results compared to [9]. This Duffing oscillator is given by

$$
m \ddot{x}+d \dot{x}+c x+\alpha x^{3}=F_{0} \cos \Omega t
$$

with $m$ being the oscillator mass, $d$ the damping coefficient, $c$ the linear stiffness, $\alpha$ the coefficient of the nonlinear stiffness, $F_{0}$ the excitation force amplitude and $\Omega$ the circular excitation frequency. In the case of the softening Duffing oscillator $\alpha$ is negative, while $m, d, c$ and $F_{0}$ are positive. This equation is transformed with respect to dimensionless time derivatives by introducing the circular frequency of the undamped free linear vibrations $\omega_{0}=\sqrt{c / m}$, the damping ratio $D=d /(2 \sqrt{c m})$ and the dimensionless time $\tau=\omega_{0} t$ as

$$
x^{\prime \prime}(\tau)+2 D x^{\prime}(\tau)+x(\tau)+\varepsilon x^{3}(\tau)=f \cos (\eta \tau)
$$

with ()$^{\prime}=\mathrm{d}() / \mathrm{d} \tau, \varepsilon=\alpha /\left(m \omega_{0}^{2}\right), f=F_{0} /\left(m \omega_{0}^{2}\right)$ and $\eta=\Omega / \omega_{0}$. This equation 2 2 shall now be solved approximately by using Harmonic Balance with the ansatz

$$
x(\tau)=\sum_{k=1}^{n}\left(a_{k} \cos (k \eta \tau)+b_{k} \sin (k \eta \tau)\right) .
$$

It should be emphasized that, as long as nothing else is stated, for the examples shown in this paper, only odd $k$ are considered, as we have in general cubic nonlinearities. This means that $a_{k}=b_{k}=0$ for even $k$ in this case. 
Introducing (3) into (2) results in a set of nonlinear algebraic equations

$$
\sum_{k=1}^{n}\left(\tilde{a}_{k} \cos (k \eta \tau)+\tilde{b}_{k} \sin (k \eta \tau)\right)=f \cos (\eta \tau)-\sum_{k=n+1}^{3 n}\left(\tilde{a}_{k} \cos (k \eta \tau)+\tilde{b}_{k} \sin (k \eta \tau)\right) .
$$

Herein the coefficients $\tilde{a}_{k}, \tilde{b}_{k}$ are nonlinear functions of the ansatz coefficients $a_{k}, b_{k}$. In the method of Harmonic Balance, the higher order frequency terms

$$
\sum_{k=n+1}^{3 n}\left(\tilde{a}_{k} \cos (k \eta \tau)+\tilde{b}_{k} \sin (k \eta \tau)\right)
$$

in (4) are neglected, i.e. not considered for the calculation of the coefficients $a_{k}, b_{k}$. This allows the calculation of the coefficients $a_{k}, b_{k}$ from the thereby modified equation (4) by balancing the respective harmonics.

For visualizing the results $x(\tau), \hat{x}$ is defined as

$$
\hat{x}=\max _{0 \leq \tau \leq \frac{2 \pi}{\eta}}\left\{\sum_{k=1}^{n}\left(a_{k} \cos (k \eta \tau)+b_{k} \sin (k \eta \tau)\right)\right\} .
$$

Now as mentioned before an error criterion is introduced by considering the neglected terms in the Harmonic Balance method ([3], [9], [10], [11]). The corresponding coefficients $\tilde{a}_{k}, \tilde{b}_{k}$ of the neglected higher order terms can be calculated using the solution for $a_{k}, b_{k}$ and the error $\hat{e}$ is defined from this by

$$
\hat{e}=\max _{0 \leq \tau \leq \frac{2 \pi}{\eta}}\left\{\sum_{k=n+1}^{3 n}\left(\tilde{a}_{k} \cos (k \eta \tau)+\tilde{b}_{k} \sin (k \eta \tau)\right)\right\} .
$$

Finally the relative error $\tilde{e}$ is introduced as

$$
\tilde{e}=\frac{\hat{e}}{\hat{x}} \text {. }
$$

This relative error is the main evidence for considering a solution as an artifact. If $\hat{e}$ remains larger than $1 \%$ even for increasing numbers of ansatz orders $n$, the solution is considered to be an artifact. According to our experience, this is accompanied by changing of the shape of the respective solutions with increasing ansatz orders and non-convergence of the coeffcients $a_{k}$ and $b_{k}$ which can be used as additional attributes.

With respect to the demonstration of some results, the parameters

$$
D=0.08, \varepsilon=-0.1 \text { and } f=0.2
$$

are taken. The results are displayed by $\hat{x}(6)$. Here and in the following a classification of the solutions is done in that way, that the solutions are divided in solutions with relative errors $\tilde{e}$ larger $1 \%$ and solutions with relative errors $\tilde{e}$ lower $1 \%$. The former are sketched with red color. Solutions with relative error lower than $1 \%$ are investigated via Floquet theory for stability and corresponding results are sketched in grey in the case of unstable and blue in the case of asymptotically stable solutions. 

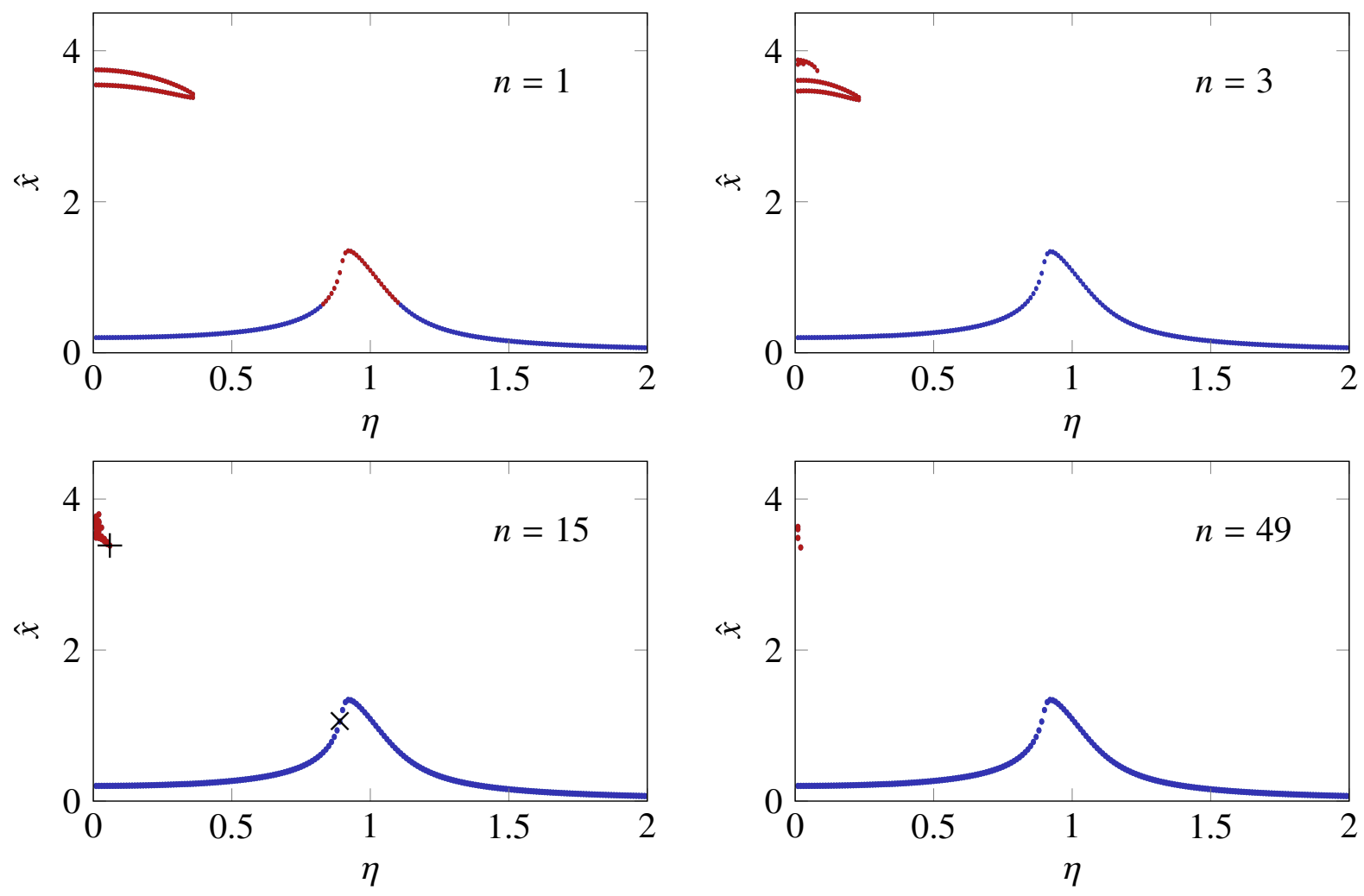

Figure 1: Solutions $\hat{x}$ according to 6 for the Duffing oscillator 2 with Harmonic Balance ansatz 3 in case of parameters $D=0.08, \varepsilon=-0.1$ and $f=0.2$. Solutions with relative error $\tilde{e}$ according to 8 larger than $1 \%$ are sketched in red color. Solutions with relative error $\tilde{e}$ lower than $1 \%$ are sketched in grey color in the unstable case (not present in these figures) and blue color in the asymptotically stable case. The coefficients $a_{k}$ and $b_{k}$ of the solutions marked by "x" and "+" (downside left) are listed in tables 1-4.

Corresponding results are shown in Figure 1 for increasing ansatz orders $n$. There are two types of solutions. First the well-known resonance curve starting for small $\eta$ with also small $\hat{x}$, having the resonance peak close to $\eta=1$ and going to zero for $\eta \rightarrow \infty$. Due to the softening characteristic, the resonance peak is turning to the left. This solution preserves its basic shape for all ansatz orders. With the error analysis it can be seen, that this solution shows very small relative errors $\tilde{e}$ being smaller than $1 \%$ for $n \geq 3$. In the chosen parameter set, the damping is too large to have a region of three coexisting solutions close to resonance.

The other solution is the "nose-like" one occurring for small $\eta$ and large $\hat{x}$. For $n=1$, this solution can be found sketched in many textbooks on nonlinear dynamics. It can also occur in case of using perturbation analysis [9]. Increasing the ansatz order, it changes its shape and vanishes more and more, while the errors of the remaining parts are still larger than $1 \%$. Therefore we consider this solution to be an artifact solution. The change of solution shape is also characteristic for these artifacts according to our 
experience.

It shall be mentioned, that the error considered by this method is not an error of comparing an exact solution with the approximate solution but an error in fulfilling the equation of motion. As a second criterion, also the convergence of the cosine and sine coefficients $a_{k}$ and $b_{k}$ can be considered, as it is done also e.g. in [14]. This is shown in the following.

In Tables 1 and 2 the coefficients $a_{k}$ and $b_{k}$ for the solutions marked in case $n=15$ in Figure 1 by " $x$ " for $\eta=0.89$ are shown. In this case the used error criterion gives a small error as result and in fact convergence of the coefficients can be observed.

This changes in the case of the solution marked in case $n=15$ in Figure 1 by "+" for $\eta=0.06$. Here a large relative error $\tilde{e}$ is observed and in fact Tables 3 and 4 show a non-convergence of the coefficients $a_{k}$ and $b_{k}$.

It shall be additionally mentioned that in [9] it was furthermore shown, that an additional unstable solution (not being an artifact) with non-zero mean value can be calculated also for small $\eta$. To get this solution as a result of Harmonic Balance, the ansatz (3) has to be extended by a constant term $a_{0}$. We will see in one of the upcoming examples, that also coefficients $a_{k}, b_{k}$ with even $k$ have to be taken into consideration despite the fact, that there are only cubic nonlinearities in the equation of motion, as it was also e.g. observed in [15].

\section{Extended Duffing oscillator}

As next example we consider an extended Duffing oscillator

$$
x^{\prime \prime}+2 D x^{\prime}+x+\varepsilon_{1} x^{3}+\varepsilon_{2} x^{2} x^{\prime}=f_{1} \cos (\eta \tau)+f_{2} x^{2} \cos (\eta \tau)
$$

with the same denominations and the same methods as introduced in section 2 , The cubic nonlinearity with parameter $\varepsilon_{1}$ is complemented by a cubic damping term $x^{2} x^{\prime}$ with parameter $\varepsilon_{2}$ and the harmonic excitation with intensity $f_{1}$ is combined with a nonlinear parameter excitation term with constant $f_{2}$. Such an equation can e.g. be obtained from piezoceramic continua by adding conservative and non-conservative terms in piezoelectric coupling and elasticity as described in [16]. The parameters here are chosen arbitrarily to show certain nonlinear phenomena and do not necessarily represent a real piezoceramic.

Let's first consider the "good-natured" parameter set $D=0.08, \varepsilon_{1}=0.0, \varepsilon_{2}=$ $-0.1, f_{1}=0.1$ and $f_{2}=0.1$. Using again the ansatz (3) with only odd $k, \hat{x}$ can be calculated according to equation (6) and the relative error $\tilde{e}$ corresponding to equation (8). The results are shown in Figure 2 As $\varepsilon_{1}=0.0$, the system shows neither a stiffening nor a softening character. As shown in [10], [11] there is an "island" solution, that shall be considered in the following. In fact the analysis shows, that the island is not an artifact, but consists of unstable solutions. The same characteristics for nonartifact solutions as in the section before can be observed, that the low-error solutions keep their shape and that there is convergence with respect to the stability behavior. In fact there is no visible change between the cases $n=5$ and $n=49$ for this parameter set. 

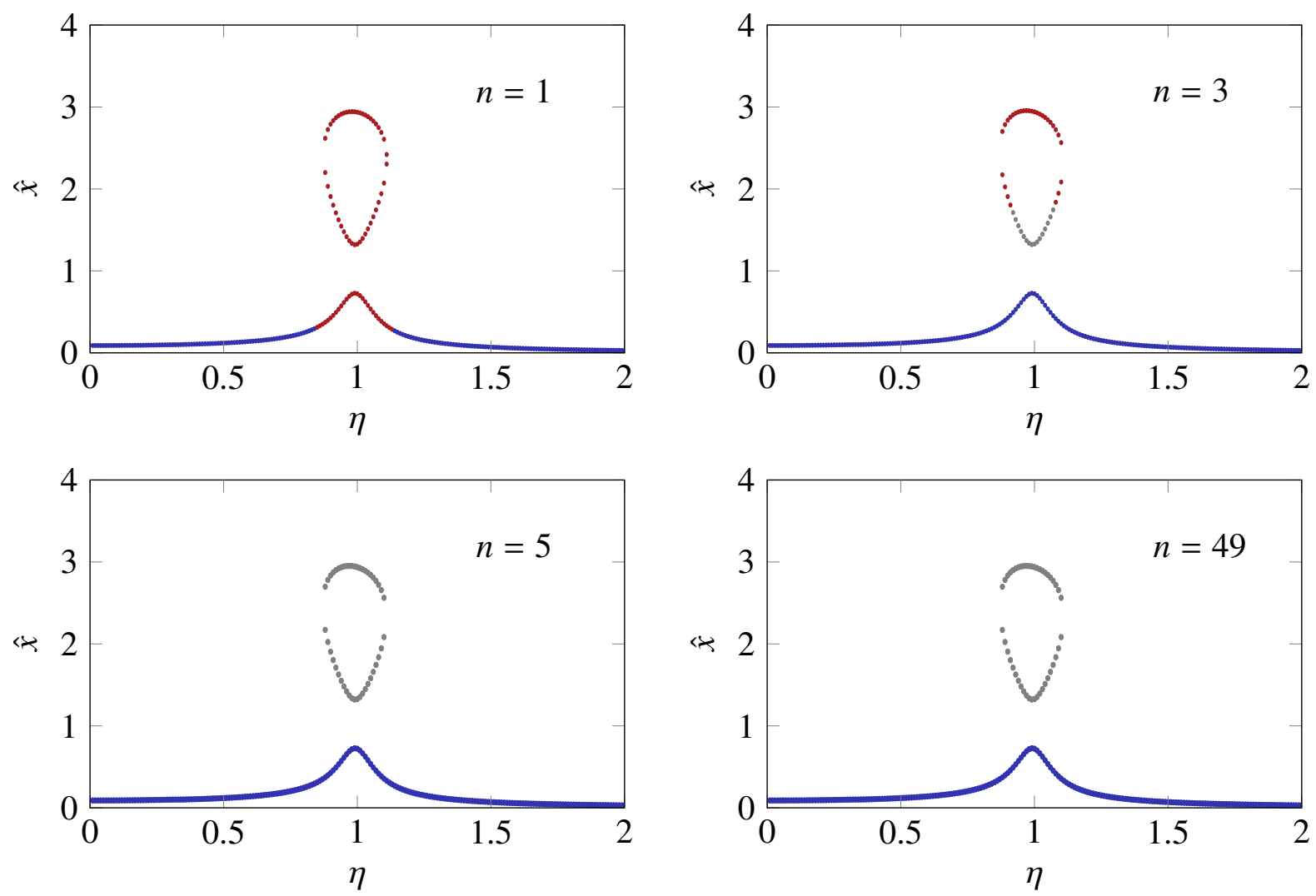

Figure 2: Solutions $\hat{x}$ according to 6 for the extended Duffing oscillator 10 with Harmonic Balance ansatz (3) in case of parameters $D=0.08, \varepsilon_{1}=0.0, \varepsilon_{2}=-0.1, f_{1}=0.1$ and $f_{2}=0.1$. Solutions with relative error $\tilde{e}$ according to 8 larger than $1 \%$ are sketched in red color. Solutions with relative error $\tilde{e}$ lower than $1 \%$ are sketched in grey color in the unstable case and blue color in the asymptotically stable case.

Less "good-natured" is the parameter set $D=0.08, \varepsilon_{1}=-0.4, \varepsilon_{2}=0, f_{1}=$ 0.1 and $f_{2}=0.1$. Corresponding results are displayed in Figure 3. For $n=1$ this oscillator shows a similar behavior to the classic Duffing oscillator in case of Harmonic Balance solution with $n=1$ as shown in Figure 1 In this earlier case the "nose" solution is identified as an artifact while here an increasing ansatz order shows another picture. In fact the right part of the "nose" keeps its shape and the relative errors are moving below the $1 \%$ border. Most of this low relative error part shows instability while for increasing orders of $n$ also a small asymptotically stable part can be identified. A similar behavior is also possible for the classic Duffing oscillator as shown in [12] and [10]. As can be seen in Figure 3 the left part of the "nose" maintains its large errors and therefore is considered to be an artifact. Overall also for the non-artifact parts, higher ansatz orders are necessary to get convergence compared to the examples before.

In all examples so far only odd $k$ were considered in the ansatz (3). Based on the 

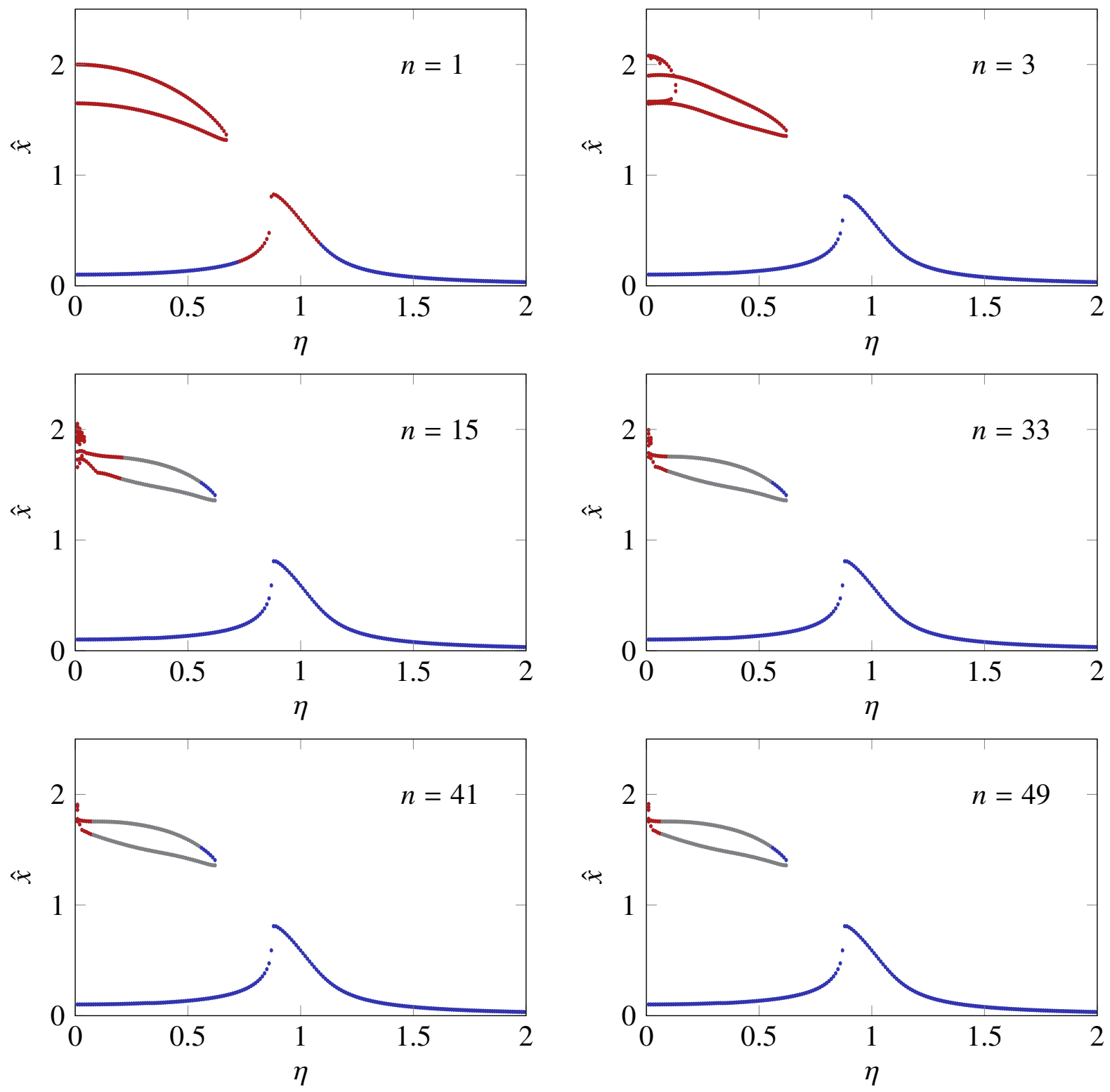

Figure 3: Solutions $\hat{x}$ according to 6 for the extended Duffing oscillator 10 with Harmonic Balance ansatz (3) in case of parameters $D=0.08, \varepsilon_{1}=-0.4, \varepsilon_{2}=0, f_{1}=0.1$ and $f_{2}=0.1$. Solutions with relative error $\tilde{e}$ according to $(8)$ larger than $1 \%$ are sketched in red color. Solutions with relative error $\tilde{e}$ lower than $1 \%$ are sketched in grey color in the unstable case and blue color in the asymptotically stable case.

experience from [9], that even in the case of a softening Duffing oscillator with purely 

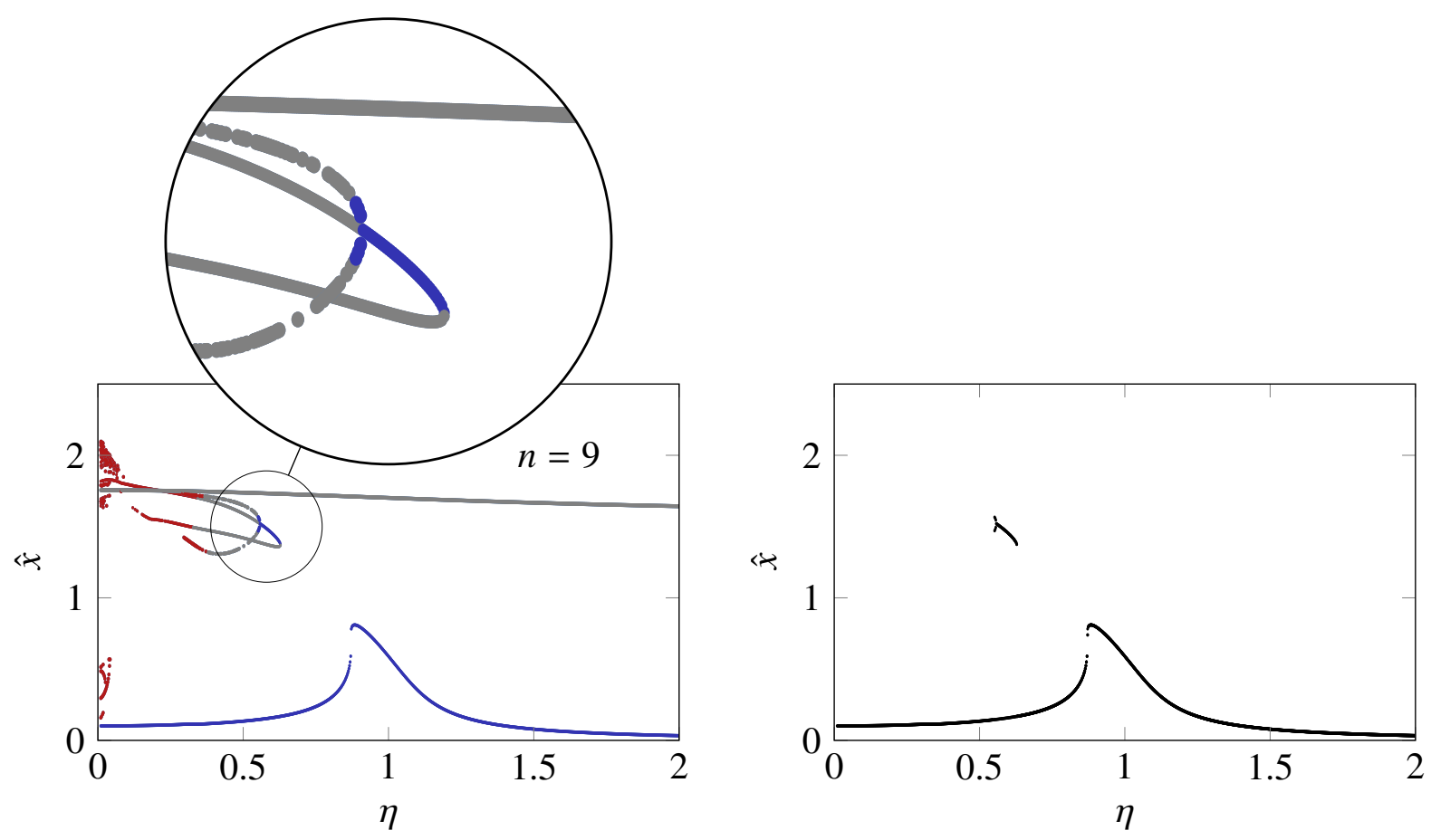

Figure 4: Same problem and parameter set as in Figure 3 Harmonic Balance solution taking also into account a nonzero mean value $a_{0}$ and $a_{k}, b_{k}$ with even $k$ (left). Result from numerical integration for comparison (right)

cubic nonlinearity, solutions with nonzero mean value occur, the ansatz is extended as

$$
x(\tau)=a_{0}+\sum_{k=1}^{n}\left(a_{k} \cos (k \eta \tau)+b_{k} \sin (k \eta \tau)\right)
$$

taking now into consideration a constant $a_{0}$ but also even $k$. Corresponding results are shown in Figure 4 with the results for the proposed Harmonic Balance method (left) for $n=9$. First, there is an additional almost horizontal branch of unstable solutions. They are similar to those described in [9], namely forced vibrations with nonzero mean value around the unstable static equilibrium displacements resulting from the softening Duffing characteristic. Compared to the earlier results in Figure 3 there are also two small branches bifurcating from the stable part of the "nose". In fact it can be observed, that these solutions contain symmetry breaking even parts in the Harmonic Balance. Both the existence of the stable part of the "nose" and the bifurcating asymmetric solutions could be confirmed by numerical integration as can be seen in figure 4 (right). This demonstrates again, that some a priori knowledge is indispensable when applying the Harmonic Balance method.

Some additional comments shall be given at the end of this chapter. While in this paper only softening or neutral cases, i.e. $\varepsilon<0$ in equation(2) and $\varepsilon_{1} \leq 0$ in 
equation 10 have been investigated, a stiffening case was considered in [11] in case of equation(10), with the parameter set $\varepsilon_{1}=0.1, \varepsilon_{2}=0.25, D=0.1, f_{1}=0.1$ and $f_{2}=0.1$. In that case the resonance peak is - as can be expected - turning to the right, and an additional non-artifact unstable island solution like in figure 2 occurs. In future work, the stiffening case could also be interesting with respect to possible artifact or asymmetric solutions.

Looking for potential problems of the proposed method it shall be remembered, that in general in Harmonic Balance a corresponding set of nonlinear algebraic equations for the coefficients $a_{k}, b_{k}$ has to be solved. Depending on the considered system and ansatz order this can assume large proportions. It can be seen in the example from figure 3 with the (not very small but) moderate $\varepsilon_{1}=-0.4$, that the stronger nonlinearity results in slower convergence of the method. This makes higher ansatz numbers and solution of larger equation sets necessary and complicates the applicability.

\section{Conclusions}

The actual paper shows results of using an extended Harmonic Balance method applied to the classic and an extended Duffing oscillator. The Harmonic Balance method is hereby extended by an error criterion evaluating the neglected terms and comparing them with the approximate solution. All solutions calculated are classified in the figures in three types in that way, that solutions with relative errors larger $1 \%$ are considered and solutions with relative errors lower $1 \%$ are divided in asymptotically stable and unstable solutions. Solutions showing large relative errors even for high ansatz orders are then considered to be artifacts. According to our experience, non-artifact solutions are accompanied by converging coefficients in the expansion. On the other hand in the examples investigated so far, solutions with high relative errors change their shapes for increasing ansatz orders and show non-converging coefficients. The low-error solutions are examined for their stability using Floquet's theory. The examples considered in this paper showed solutions like "noses" and "islands" and some of them could be identified as artifacts. On the other hand for increasing ansatz order, parts of "noses" or even complete "islands" could be detected to show low errors for increasing ansatz orders. Some parts are even stable what could be confirmed also by numerical integration in time. Even for purely cubic nonlinearities, there exist asymmetric solutions with non-vanishing mean value and coefficients $a_{k}, b_{k}$ for even $k$ as could be seen in the last example. Compared to numerical integration, the proposed Harmonic Balance procedure offers to obtain a faster calculation of existing multiple solutions and their character.

\section{References}

[1] P. Hagedorn, Nichtlineare Schwingungen, Akademische Verlagsgesellschaft, Wiesbaden, 1978.

[2] T. Hill, S. Neild, D. Wagg, Comparing the direct normal form method with harmonic balance and the method of multiple scales, Procedia Engineering (199) (2017) $869-874$. 
[3] M. Urabe, A. Reiter, Numerical computation of nonlinear forced oscillations by Galerkin's procedure, Journal of Mathematical Analysis and Applications (14) (1966) 107-140.

[4] M. Chowdhury, M. Hosen, K. Ahmad, M. Ali, A. Ismail, High-order approximate solutions of strongly nonlinear cubic-quintic Duffing oscillator based on the harmonic balance method, Results in Physics, (14) (2017) 3962 - 3967.

[5] M. Saifur, A. Hasan, Modified harmonic balance method for the solution of nonlinear jerk equations, Results in Physics (8) (2018) 893 - 897.

[6] G. Liu, Z. R. Lv, J. K. Liu, Y. Chen, Quasi-periodic aeroelastic response analysis of an airfoil with external store by incremental harmonic balance method, International Journal of Non-Linear Mechanics (100) (2018) 10 - 19.

[7] H. Xiong, X. Kong, H. Li, Z. Yang, Vibration analysis of nonlinear systems with the bilinear hysteric oscillator by using incremental harmonic balance method, Communications in Nonlinear Science and Numerical Simulation (42) (2017) 437 -450 .

[8] I. Kovacic, M. Brennan, The Duffing Equation: Nonlinear Oscillators and their Behaviour, John Wiley \& Sons, Ltd, 2011.

[9] U. von Wagner, L. Lentz, On some aspects of the dynamic behavior of the softening Duffing oscillator under harmonic excitation, Archive of Applied Mechanics 8 (86) (2016) 1383-1390.

[10] U. von Wagner, L. Lentz, On artifacts in nonlinear dynamics, Vibration, Control and Stability of Dynamical Systems, Proceedings of DSTA (2017) 525-535.

[11] U. von Wagner, L. Lentz, On artifact solutions of semi-analytic methods in nonlinear dynamics, Archive of Applied Mechanics (2018) doi 10.1007/s00419-018$1397-3$.

[12] van Dooren R., On the transition form regular to chaotic behaviour in the Duffing oscillator, Journal of Sound and Vibration 2 (123) (1988) 327-339.

[13] A. Ferri, M. J. Leamy, Error estimates for harmonic-balance solutions of nonlinear dynamical systems, Proceedings of 50th AIAA/ASME/ASCE/AHS/ASC Structures, Structural Dynamics, and Materials Conference (2009) AIAA 20092667.

[14] L. Liu, J. Thomas, E. Dowell, P. Attar, K. Hall, A comparison of classical and high dimensional harmonic balance approaches for a Duffing oscillator, Journal of Computational Physics 1 (215) (2006) 298 - 320.

[15] A. H. Nayfeh, H. E. Sanchez, Bifurcations in a forced softening Duffing oscillator, International Journal of Non-Linear Mechanics 24 (6) (1989) 483 - 497.

[16] S. K. Parashar, U. von Wagner, P. Hagedorn, Nonlinear shear-induced flexural vibrations of piezoceramic actuators: experiments and modeling, Journal of Sound and Vibration (285) (2005) 989-1014. 
Table 1: coefficients $a_{k}$ of the solution marked by "x" in Figure $11 \eta=0.89$

\begin{tabular}{r|rrrrrrrr}
$\mathrm{n}$ & $a_{1}$ & $a_{3}$ & $a_{5}$ & $a_{7}$ & $a_{9}$ & $a_{11}$ & $a_{13}$ & $a_{15}$ \\
\hline 1 & 0.69512 & 0 & 0 & 0 & 0 & 0 & 0 & 0 \\
3 & 0.69570 & 0.00373 & 0 & 0 & 0 & 0 & 0 & 0 \\
5 & 0.69570 & 0.00373 & -0.00001 & 0 & 0 & 0 & 0 & 0 \\
7 & 0.69570 & 0.00373 & -0.00001 & 0 & 0 & 0 & 0 & 0 \\
9 & 0.69570 & 0.00373 & -0.00001 & 0 & 0 & 0 & 0 & 0 \\
11 & 0.69570 & 0.00373 & -0.00001 & 0 & 0 & 0 & 0 & 0 \\
13 & 0.69570 & 0.00373 & -0.00001 & 0 & 0 & 0 & 0 & 0 \\
15 & 0.69570 & 0.00373 & -0.00001 & 0 & 0 & 0 & 0 & 0
\end{tabular}

Table 2: coefficients $b_{k}$ of the solution marked by "x" in Figure $11 \eta=0.89$

\begin{tabular}{r|rrrrrrrr}
$\mathrm{n}$ & $b_{1}$ & $b_{3}$ & $b_{5}$ & $b_{7}$ & $b_{9}$ & $b_{11}$ & $b_{13}$ & $b_{15}$ \\
\hline 1 & 0.80206 & 0 & 0 & 0 & 0 & 0 & 0 & 0 \\
3 & 0.79688 & -0.00284 & 0 & 0 & 0 & 0 & 0 & 0 \\
5 & 0.79687 & -0.00284 & -0.00002 & 0 & 0 & 0 & 0 & 0 \\
7 & 0.79687 & -0.00284 & -0.00002 & 0 & 0 & 0 & 0 & 0 \\
9 & 0.79687 & -0.00284 & -0.00002 & 0 & 0 & 0 & 0 & 0 \\
11 & 0.79687 & -0.00284 & -0.00002 & 0 & 0 & 0 & 0 & 0 \\
13 & 0.79687 & -0.00284 & -0.00002 & 0 & 0 & 0 & 0 & 0 \\
15 & 0.79687 & -0.00284 & -0.00002 & 0 & 0 & 0 & 0 & 0
\end{tabular}

Table 3: coefficients $a_{k}$ of the solution marked by " + " in Figure $11 \eta=0.06$

\begin{tabular}{r|rrrrrrrr}
$\mathrm{n}$ & $a_{1}$ & $a_{3}$ & $a_{5}$ & $a_{7}$ & $a_{9}$ & $a_{11}$ & $a_{13}$ & $a_{15}$ \\
\hline 1 & -3.67914 & 0 & 0 & 0 & 0 & 0 & 0 & 0 \\
3 & -3.78828 & 0.59545 & 0 & 0 & 0 & 0 & 0 & 0 \\
5 & -3.62553 & 0.19122 & 0.40248 & 0 & 0 & 0 & 0 & 0 \\
7 & -3.34890 & -0.33538 & 0.64898 & -0.15432 & 0 & 0 & 0 & 0 \\
9 & -2.99132 & -0.82318 & 0.54249 & 0.24145 & -0.23127 & 0 & 0 & 0 \\
11 & -2.56463 & -1.16502 & 0.12736 & 0.44813 & 0.08710 & -0.17347 & 0 & 0 \\
13 & -2.06646 & -1.28601 & -0.39000 & 0.20482 & 0.31504 & 0.12752 & -0.06258 & 0 \\
15 & -1.46211 & -1.11565 & -0.71877 & -0.30981 & -0.00469 & 0.14619 & 0.16174 & 0.10051
\end{tabular}

Table 4: coefficients $b_{k}$ of the solution marked by " + " in Figure $1, \eta, \eta=0.06$

\begin{tabular}{r|rrrrrrrr}
$\mathrm{n}$ & $b_{1}$ & $b_{3}$ & $b_{5}$ & $b_{7}$ & $b_{9}$ & $b_{11}$ & $b_{13}$ & $b_{15}$ \\
\hline 1 & 0.67137 & 0 & 0 & 0 & 0 & 0 & 0 & 0 \\
3 & 1.24476 & -0.87185 & 0 & 0 & 0 & 0 & 0 & 0 \\
5 & 1.79309 & -1.17016 & 0.41445 & 0 & 0 & 0 & 0 & 0 \\
7 & 2.29957 & -1.19090 & 0.04369 & 0.34652 & 0 & 0 & 0 & 0 \\
9 & 2.75390 & -0.95800 & -0.42070 & 0.34839 & 0.13896 & 0 & 0 & 0 \\
11 & 3.15069 & -0.52464 & -0.69577 & -0.02656 & 0.28563 & 0.09942 & 0 & 0 \\
13 & 3.48854 & 0.03696 & -0.60558 & -0.41711 & -0.02654 & 0.17824 & 0.13803 & 0 \\
15 & 3.76891 & 0.65178 & -0.11999 & -0.36018 & -0.32780 & -0.17952 & -0.03047 & 0.05862
\end{tabular}

